\title{
Nível de evidência dos estudos relacionados à ansiedade, estresse e depressão dos profissionais de enfermagem
}

\author{
Evidence level of studies related to anxiety, stress and depression of nursing professionals \\ Nivel de prueba de estudios relacionados con ansiedad, estrés y depresión de profesionales de \\ enfermería
}

Recebido: 17/12/2020 | Revisado: 26/12/2020 | Aceito: 28/12/2020 | Publicado: 02/01/2021

\author{
Camila Cristina Machado Melo \\ ORCID: https://orcid.org/ 0000-0002-3220-1515 \\ Universidade do Estado de Minas Gerais, Brasil \\ E-mail: camila.2104925@discente.uemg.br \\ Luiz Felipe Bernardes \\ ORCID: https://orcid.org/ 0000-0002-5694-5682 \\ Universidade do Estado de Minas Gerais, Brasil \\ E-mail: luiz.2122139@discente.uemg.br \\ Glilciane Morceli \\ ORCID: https://orcid.org/0000-0001-8216-9931 \\ Universidade do Estado de Minas Gerais, Brasil \\ E-mail: glilciane.morceli@uemg.br \\ Policardo Gonçalves da Silva \\ ORCID: https://orcid.org/0000-0001-9095-6409 \\ Universidade do Estado de Minas Gerais, Brasil \\ E-mail: policardo.silva@uemg.br \\ Sandra de Souza Pereira \\ ORCID: https://orcid.org/ 0000-0002-1918-7771 \\ Universidade do Estado de Minas Gerais, Brasil \\ E-mail: sandra.pereira@uemg.br \\ Sérgio Valverde Marques dos Santos \\ ORCID: https://orcid.org/0000-0001-9412-9515 \\ Universidade do Estado de Minas Gerais, Brasil \\ E-mail: sergiovalverdemarques@ hotmail.com
}

\begin{abstract}
Resumo
Objetivo: Identificar os níveis de evidência dos estudos relacionados à ansiedade, estresse e depressão entre profissionais de enfermagem. Método: Revisão integrativa da literatura, realizada nas bases/bancos de dados LILACS, BVSENF e SCIELO, entre 2015 e 2019, com os descritores: enfermagem, ansiedade, estresse e depressão. Para realizar a busca bibliográfica adotou-se a estratégia PICO, para selecionar e avaliar os artigos, utilizou-se a estratégia recomendada pelo grupo PRISMA. Para verificar o nível de evidência dos estudos, utilizou-se o instrumento recomendado por Gershon e colaboradores (1999). Resultados: Foram encontrados 525 artigos, destes apenas 17 atenderam aos critérios de inclusão. Dos artigos selecionados, a maioria foi publicado entre 2018 e 2019 (53\%). Destes, grande parte possui o delineamento de estudo descritivo e quantitativo (47\%). Destaca-se que apenas 30\% dos estudos foram realizados em hospitais universitários e a maioria na rede pública. A maioria dos estudos buscaram evidências sobre o estresse no ambiente de trabalho dos profissionais de enfermagem (94\%). Apenas $29 \%$ dos estudos investigaram a ansiedade e 17\% a depressão. Constatou-se que os estudos apresentaram fracos níveis de evidência, uma vez que todos eles tiveram abordagem descritiva, considerada como nível 6 de evidência. Conclusão: Evidenciou-se que os profissionais de enfermagem sofrem recorrentemente com ansiedade, estresse e depressão nos ambientes hospitalares. No entanto, os estudos apresentam nível de evidência fraca, apontando a necessidade de mais investigações com estudo que apresentem evidências mais fortes.
\end{abstract}

Palavras-chave: Enfermagem; Ansiedade; Estresse; Depressão; Saúde do trabalhador.

\section{Abstract}

Objective: To identify the levels of evidence from studies related to anxiety, stress and depression among nursing professionals. Method: integrative literature review, carried out in LILACS, BVSENF and SCIELO databases / databases, between 2015 and 2019, with the descriptors: nursing, anxiety, stress and depression. To carry out the bibliographic search, the PICO strategy was adopted, to select and evaluate the articles, the strategy recommended by the PRISMA group was used. To verify the level of evidence of the studies, the instrument recommended by Gershon and collaborators (1999) was used. Results: 525 articles were found, of which only 17 met the inclusion criteria. Of 
the selected articles, most were published between 2018 and 2019 (53\%). Of these, a large part has a descriptive and quantitative study design (47\%). It is noteworthy that only $30 \%$ of the studies were carried out in university hospitals and the majority in the public network. Most studies sought evidence of stress in the work environment of nursing professionals (94\%). Only $29 \%$ of studies investigated anxiety and $17 \%$ depression. It was found that the studies presented weak levels of evidence, since all of them had a descriptive approach, considered as level 6 of evidence. Conclusion: It became evident that nursing professionals suffer recurrently from anxiety, stress and depression in hospital environments. However, studies have a low level of evidence, pointing to the need for further investigations with studies that present stronger evidence.

Keywords: Nursing; Anxiety; Stress; Depression; Worker's health.

\section{Resumen}

Objetivo: Identificar los niveles de evidencia de estudios relacionados con la ansiedad, el estrés y la depresión entre profesionales de enfermería. Método: revisión integradora de la literatura, realizada en bases de datos / bases de datos LILACS, BVSENF y SCIELO, entre 2015 y 2019, con los descriptores: enfermería, ansiedad, estrés y depresión. Para realizar la búsqueda bibliográfica se adoptó la estrategia PICO, para la selección y evaluación de los artículos se utilizó la estrategia recomendada por el grupo PRISMA. Para verificar el nivel de evidencia de los estudios se utilizó el instrumento recomendado por Gershon y colaboradores (1999). Resultados: Se encontraron 525 artículos, de los cuales solo 17 cumplieron con los criterios de inclusión. De los artículos seleccionados, la mayoría fueron publicados entre 2018 y 2019 (53\%). De estos, una gran parte tiene un diseño de estudio descriptivo y cuantitativo (47\%). Cabe destacar que solo el $30 \%$ de los estudios se realizaron en hospitales universitarios y la mayoría en la red pública. La mayoría de los estudios buscaron evidencia de estrés en el ambiente laboral de los profesionales de enfermería (94\%). Solo el 29\% de los estudios investigaron la ansiedad y el 17\% la depresión. Se encontró que los estudios presentaron niveles de evidencia débiles, ya que todos tenían un enfoque descriptivo, considerado como nivel de evidencia 6. Conclusión: Se evidenció que los profesionales de enfermería sufren de forma recurrente ansiedad, estrés y depresión en el ámbito hospitalario. Sin embargo, los estudios tienen un bajo nivel de evidencia, lo que apunta a la necesidad de realizar más investigaciones con estudios que presenten evidencia más sólida.

Palabras clave: Enfermería; Ansiedad; Estrés; Depresión; Salud del trabajador.

\section{Introdução}

Os transtornos mentais são condições clínicas que podem ser caracterizadas por mudanças comportamentais, alterações nos pensamentos e emoções. Além, disso, podem se manifestar por meio de comportamentos relacionados a angústia pessoal ou deterioração do funcionamento psíquico, surtindo efeitos deletérios, atingindo não somente o indivíduo mais todos os familiares, o trabalho e sua comunidade. Estima-se que $10 \%$ dos adultos apresentam tais condições e, $25 \%$ da população mundial se manifesta, pelo menos, um transtorno mental ao longo da vida (Carvalho et al., 2016)

A Organização Mundial de Saúde estimou que 36\% da população brasileira pode desenvolver algum tipo de transtorno mental em algum momento de sua vida. Os transtornos mentais representam no mundo quatro das dez principais causas de incapacidade e afetam $25 \%$ da população em alguma fase de sua vida. No Brasil no ano de 2011, de acordo com dados coletados pelo Ministério da Previdência Social, os transtornos mentais e comportamentais foram à terceira causa de concessão de auxilio-doença por incapacidade laboral. Portanto verificou-se uma tendência de aumento, pois em 2009, foram concedidos 169.739 auxílios, em 2010, 180.986 e em 2011, 191.367 (Carlotto, 2016).

Os transtornos como depressão e o estresse estão no topo da lista dos Transtornos Mentais Comuns (TCM) mais acometidos na população. Assim, cerca de 350 milhões de pessoas, ou seja, 5\% da população mundial, sofre por depressão. Calcula-se que em 2020 a depressão seja a segunda maior causa de incapacitações no planeta. No Brasil a depressão atinge $10 \%$ da população (Santana et al., 2016)

A ansiedade é caracterizada como um sofrimento físico e psíquico de aflição, agonia, angústia, ânsia e nervosismo, considerando um estado emocional com um futuro incerto e preciso, no qual um indivíduo se sente impotente e indefeso. Popularmente é conhecida como mecanismo de luta ou fuga, considerando sua intensidade e duração, podendo ser variável de indivíduo para indivíduo, com capacidade de resiliência e situações que eles vivenciam (Andrade et al., 2019).

A depressão é um transtorno afetivo acompanhado de alterações de humor, tristeza, vazio e irritação, bem como de alterações somáticas e cognitivas, que podem causar alterações na vida da pessoa. Este transtorno possui algumas 
particularidades, como o mal-estar individual e social. A Organização Mundial de Saúde afirma que a depressão é um transtorno mental, caracterizado pela tristeza, perda de interesse nas tarefas do cotidiano, perda do apetite ou sono e desanimo (Hajjar et al., 2017).

Já o estresse, é considerado como um estado de tensão, com ruptura de equilíbrio interno do organismo, podendo ser identificado em sua fase inicial, com diversos sinais e sintomas psicossomáticos como taquicardia, gastrite, alterações cardiovasculares, insônia e outros. Por sua natureza, o organismo sempre busca o equilíbrio, automaticamente, realizando um esforço especial ou uma resposta adaptativa para conceder a homeostase interior, provocando um considerável desgaste e utilização de reservas de energia física e mental (Oliveira et al., 2017).

Os profissionais da saúde sofrem recorrentemente com os transtornos mentais em seu trabalho. Estes trabalhadores vivenciam inúmeras situações de desgastantes na prática clínica, pois provém da frequente exposição a um ou mais elementos que favorecem o aparecimento de doenças ou de sofrimento psíquico. Esses transtornos podem afetar negativamente nos resultados do trabalho desses profissionais e na qualidade da assistência ofertada aos usuários (Alves, 2015).

Entre os profissionais da saúde, a equipe de enfermagem é a que mais se expõe aos transtornos mentais no ambiente de trabalho. Alguns fatores que estão sempre presentes na categoria, como longas jornadas de trabalho, turnos ininterruptos e duplos vínculo, recebem destaque, pois podem levar ao desencadeamento de doenças psicossomáticas nos trabalhadores (Silva, 2017).

Além disso, atender diariamente pacientes com diferentes doenças, enfrentar a dor, o sofrimento, a morte, o excesso de trabalho, a elevada responsabilidade e atividades de plantão, também podem acarretar às doenças mentais. Para os profissionais que lidam diretamente com o atendimento em saúde mental, a vulnerabilidade à sobrecarga emocional, bem como ao medo em ser agredido e o cansaço, podem favorecer o estresse, a ansiedade, a depressão, dentre outros transtornos (Fernandes \& Marcolan, 2017).

Deste modo, justifica-se a importância de investigar os estudos sobre transtornos mentais entre os profissionais de enfermagem, bem como promover informações sobre essa temática. Isto, devido ao fato de transtornos como ansiedade, estresse e depressão estarem presente na vida e no trabalho desses profissionais. Assim, torna-se relevante identificar na literatura científica os estudos relacionados à ansiedade, estresse e depressão entre profissionais de enfermagem e verificar os níveis de evidências dessas investigações.

Para isso, objetivou-se com este estudo, identificar os níveis de evidência dos estudos relacionados à ansiedade, estresse e depressão entre profissionais de enfermagem.

\section{Metodologia}

Trata-se de uma revisão integrativa da literatura, um dos métodos de pesquisa utilizados na Prática Baseada em Evidência (PBE), permitindo a incorporação das evidências na prática clínica (Mendes et al., 2008, Souza et al., 2010).

A revisão integrativa pode ser utilizada como um instrumento gerador de conhecimento em enfermagem. Por ter uma abordagem que permite a inclusão de metodologias diversificadas, esta tem um papel importante na PBE, podendo então auxiliar o pesquisador a sumarizar literatura teórica e empírica sobre um tema específico (Souza et al., 2010).

As etapas para o desenvolvimento desse estudo foram: a identificação do tema e seleção da questão de investigação de pesquisa; estabelecimento de critérios de inclusão e exclusão de artigos (busca da literatura); definição das informações a serem extraídas dos estudos; avaliação dos dados; análise e apresentação dos resultados; e apresentação da revisão (Mendes et al., 2008).

Para direcionar o estudo e alcançar os objetivos propostos, foi elaborada a seguinte pergunta: Qual nível de evidência científica disponível na literatura sobre a ansiedade, estresse e depressão em profissionais de enfermagem? 
Para responder essa pergunta, foram adotados os seguintes critérios de inclusão: artigos completos, publicados entre os anos de 2015 e 2019, em periódicos de enfermagem presentes nas bases de dados: Literatura Latino-Americana em Ciências de Saúde (LILACS); e, nas bibliotecas virtuais: Biblioteca Virtual em Saúde e Enfermagem (BVSENF) e Scientific Electronic Library (SCIELO), nos idiomas português, inglês e espanhol e que estavam disponíveis eletronicamente. Foram excluídos os artigos de revisão integrativa, artigos publicados em livros, artigos com outras temáticas, artigos incompletos, dissertação, tese e anais de eventos.

Para atender aos critérios de busca e seleção empregados, foram utilizados Descritores em Ciências da Saúde (DECS) e utilizado o operador booleano $A N D$, formando, assim, a estratégia de busca a partir dos descritores controlados: enfermagem, ansiedade, estresse e depressão, com as seguintes combinações: enfermagem and ansiedade; enfermagem and estresse; e, enfermagem and depressão.

Para realizar a busca bibliográfica, foi adotada a estratégia PICO, representado da seguinte forma: $\mathrm{P}=$ Paciente/Problema, I = Intervenção ou indicadores, $\mathrm{C}=$ Comparação e $\mathrm{O}=$ Resultado/desfecho. Essa estratégia tem sido utilizada para construir questões de pesquisa de diferentes naturezas, provenientes da prática clínica (Santos et al., 2007).

A seleção dos artigos foi realizada por meio do título e dos resumos e aqueles selecionados foram submetidos à leitura na íntegra e à análise minuciosa. Os dados extraídos das publicações selecionadas foram transcritos para um instrumento, construído pelos autores, para atender ao objetivo do estudo. O instrumento continha as seguintes variáveis: título do artigo, autores, ano de publicação, local do estudo, periódico que publicou o artigo, objetivo, método, população/amostra, doença investigada, resultados, conclusões, nível de evidência e qualidade do estudo.

Para busca e seleção dos artigos, foi adotada a estratégia recomenda pelo grupo PRISMA, que orienta o que deve conter em um bom relato de revisão sem julgar se sua forma de elaboração foi adequada, conforme representação no fluxograma a seguir. 
Figura 1 - Fluxograma da busca e seleção dos artigos relacionados a ansiedade, estresse e depressão em profissionais de enfermagem, conforme recomendado pelo Grupo PRISMA.
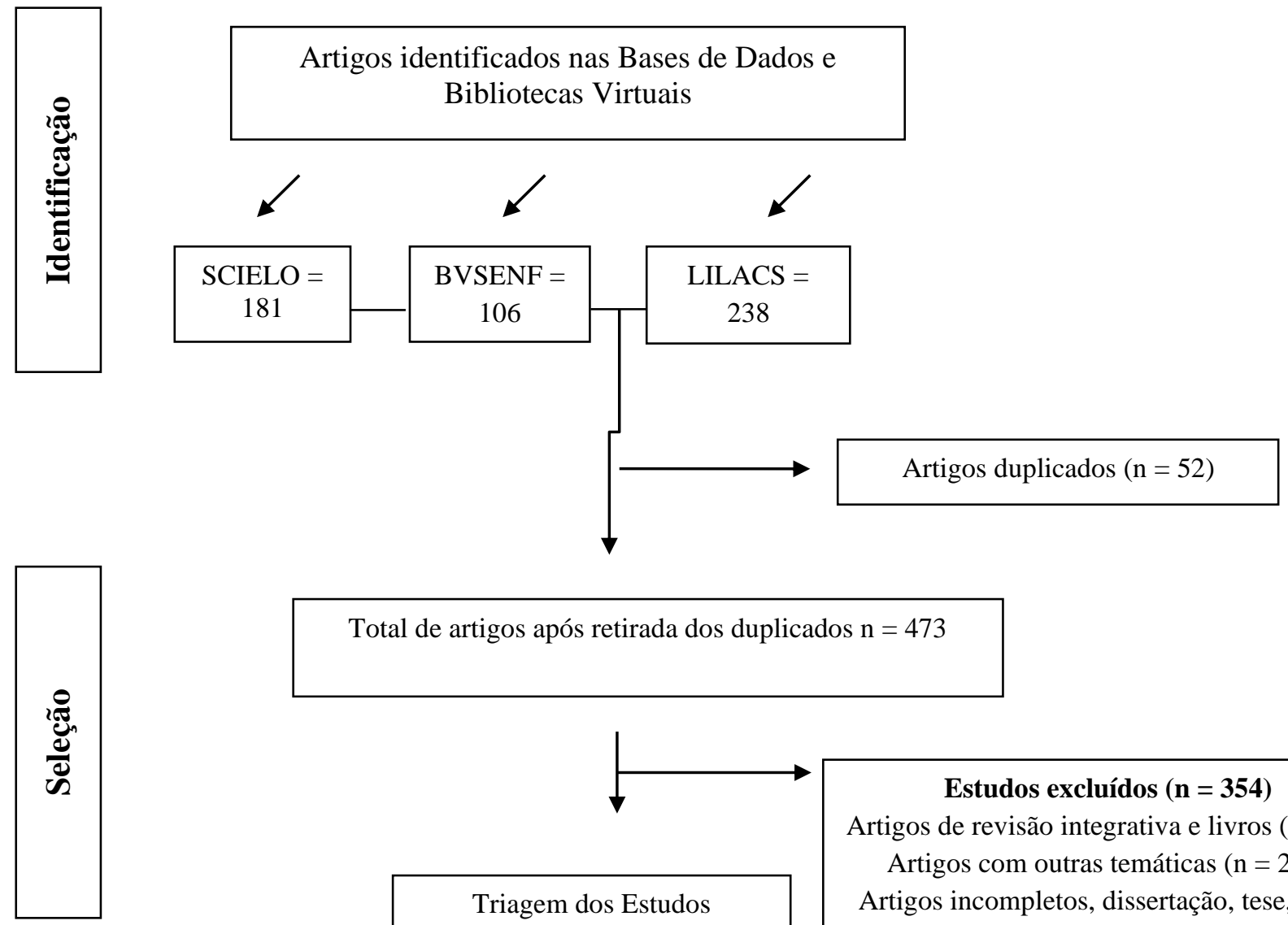

Total de artigos após retirada dos duplicados $n=473$

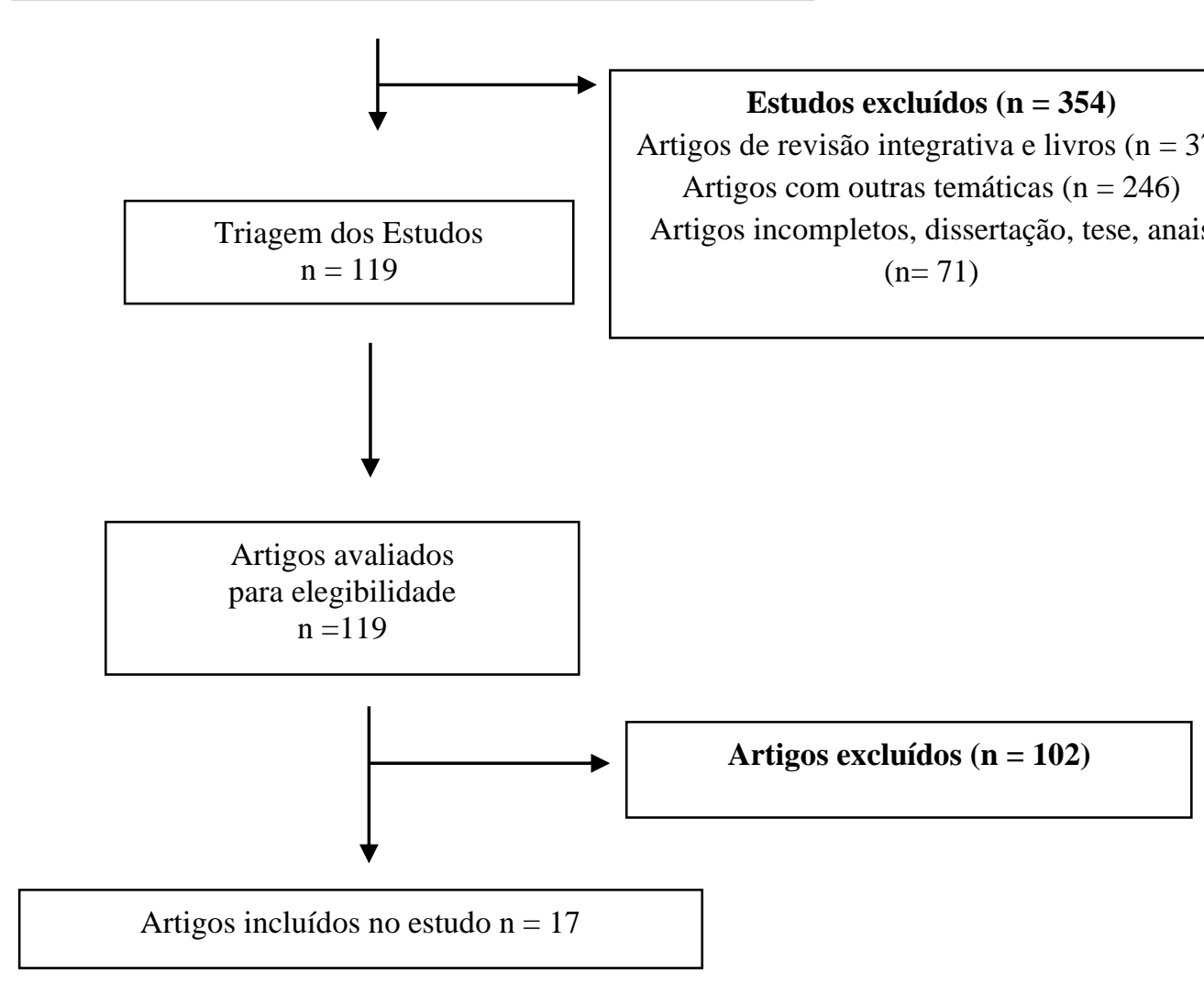

Fonte: Autores.

Adotou-se a proposta descrita por Gershon e colaboradores (1999), com o objetivo de analisar o delineamento de pesquisa e classificar os níveis de evidência científica extraídos das publicações. Para a classificação do nível de evidência, consideraram-se os níveis 1 e 2 como evidências fortes, 3 e 4 moderadas e 5 a 7 fracas (Gersho et al., 1999, Goyatá et al., 2016). 
Com relação aos aspectos éticos, por se tratar de uma revisão integrativa da literatura e pelas informações secundárias serem extraídas de artigos científicos, já publicados em bases de dados e bibliotecas virtuais, não foi necessária a autorização para utilização dos dados e nem a apreciação do Comitê de Ética em Pesquisa, conforme a Resolução Nacional 466/2012.

Os dados foram analisados de forma descritiva, com valores percentuais e absolutos e, posteriormente, apresentados em forma de tabelas e quadros.

\section{Resultados}

A busca resultou em 525 artigos, dos quais 17 estudos atenderam aos critérios de inclusão e compuseram a amostra nesta pesquisa. Foram excluídos 508 artigos, alguns estavam repetidos nas bases de dados e outros não correspondiam ao tema proposto. Logo, retratavam sobre: avaliação sobre estudantes de graduação em enfermagem, estudos com pacientes de diversas patologias, transtornos relatados em apenas algumas áreas hospitalares, pesquisa de enfermagem em saúde mental relacionada aos idosos, entre outros assuntos. Deste modo, não estavam relacionados com os transtornos mentais em profissionais da enfermagem ou não estavam disponíveis na íntegra para consulta, por via eletrônica. A Tabela 1 apresenta a descrição quantitativa dos artigos encontrados nas bases de dados, bem como os idiomas prevalentes.

Tabela 1 - Artigos identificados, selecionados e duplicados de acordo com as bases de dados e idiomas. (n = 17), Brasil, 2020.

\begin{tabular}{lcccc}
\hline Bases de dados & $\begin{array}{c}\text { Artigos } \\
\text { identificados }\end{array}$ & $\begin{array}{c}\text { Artigos } \\
\text { selecionados }\end{array}$ & $\begin{array}{c}\text { Artigos } \\
\text { duplicados }\end{array}$ & Idiomas \\
\hline SCIELO & 181 & 63 & 22 & Português e inglês \\
BVSENF & 106 & 25 & 13 & $\begin{array}{c}\text { Português } \\
\text { Português, inglês } \\
\text { e espanhol }\end{array}$ \\
LILACS & 238 & 31 & 17 & $\begin{array}{c}\text { Português, inglês } \\
\text { e espanhol }\end{array}$ \\
\hline
\end{tabular}

Fonte: Autores.

Dos artigos selecionados, $30 \%$ foram publicados no ano de 2018, 23\% em 2019, $18 \%$ em 2017, $17 \%$ em 2016 e $11 \%$ em 2015. Destes, grande parte possui o delineamento de estudo descritivo e quantitativo (47\%), bem como com delineamento transversal (36\%). O Quadro 1 apresenta a descrição dos artigos encontrados nas bases de dados, de acordo com o título, autores, delineamento e ano de publicação. 
Quadro 1 - Distribuição dos estudos de acordo com os títulos, nomes dos autores, delineamento e ano de publicação. (n = 17),

Brasil, 2020.

\begin{tabular}{|c|c|c|c|}
\hline Título & Autores & Delineamento & Ano \\
\hline $\begin{array}{l}\text { 1. Estresse ocupacional e bournout em } \\
\text { enfermeiros de um serviço de emergência: a } \\
\text { organização do trabalho }\end{array}$ & $\begin{array}{l}\text { Oliveira, E. B., Gallash, C. H., } \\
\text { Junior, P. P. A. S., Oliveira, A. V. } \\
\text { R., Valério, R. L., Dias, L. B. S. }\end{array}$ & $\begin{array}{l}\text { Pesquisa descritiva } \\
\text { e transversal }\end{array}$ & 2017 \\
\hline $\begin{array}{l}\text { 2. Estresse ocupacional e insatisfação com a } \\
\text { qualidade de vida no trabalho }\end{array}$ & $\begin{array}{l}\text { Azevedo, B. D. S., Nery, A. A., } \\
\text { Cardoso, J. P. }\end{array}$ & $\begin{array}{l}\text { Estudo descritivo } \\
\text { transversal }\end{array}$ & 2017 \\
\hline $\begin{array}{l}\text { 3. Relationship among perceived stress, } \\
\text { anxiety, depression and craniocervical pain } \\
\text { in nursing professionals under stress at } \\
\text { work. }\end{array}$ & $\begin{array}{l}\text { Pozzebon, D., Piccin, C.F., Silva, } \\
\text { A.M.T., Corrêa, E.C. R. }\end{array}$ & $\begin{array}{l}\text { Pesquisa descritiva } \\
\text { quantitativa }\end{array}$ & 2016 \\
\hline $\begin{array}{l}\text { 4. Nível de estresse em enfermeiros de uma } \\
\text { instituição hospitalar. }\end{array}$ & $\begin{array}{l}\text { Oliveira, E. M., Souza, E. A., } \\
\text { Tonini, N. S., Maraschin, M. S. }\end{array}$ & $\begin{array}{l}\text { Pesquisa de campo, } \\
\text { descritiva e } \\
\text { exploratória }\end{array}$ & 2018 \\
\hline $\begin{array}{l}\text { 5. Estresse e demais fatores de risco para } \\
\text { hipertensão arterial entre profissionais } \\
\text { militares da área de enfermagem. }\end{array}$ & $\begin{array}{l}\text { Silva, J. L. L., Lima, R. P., Taveira, } \\
\text { R. P. C., Costa, F. S., Soares, R. S. }\end{array}$ & $\begin{array}{c}\text { Pesquisa } \\
\text { quantitativa e } \\
\text { descritiva }\end{array}$ & 2016 \\
\hline $\begin{array}{l}\text { 6. Autopercepção do estresse ocupacional na } \\
\text { equipe de enfermagem de um serviço de } \\
\text { emergência. }\end{array}$ & $\begin{array}{l}\text { Silva, P. N., Silva, A., Freitas, V. } \\
\text { M., Atagiri, S., Rocha, I. C. }\end{array}$ & $\begin{array}{l}\text { Pesquisa } \\
\text { transversal, } \\
\text { descritiva, } \\
\text { exploratória e } \\
\text { quantitativa } \\
\end{array}$ & 2019 \\
\hline $\begin{array}{l}\text { 7. Estresse, coping e Burnout da equipe de } \\
\text { Enfermagem de Unidades de Terapia } \\
\text { Intensiva: fatores associados. }\end{array}$ & $\begin{array}{l}\text { Andolhe, R., Barbosa, R. L., } \\
\text { Oliveira, E. M., Costa, A. L. S., } \\
\text { Padilha, G. }\end{array}$ & $\begin{array}{c}\text { Pesquisa } \\
\text { observacional e } \\
\text { transversal }\end{array}$ & 2018 \\
\hline $\begin{array}{l}\text { 8. Características do trabalho e estresse } \\
\text { ocupacional entre enfermeiros hospitalares. }\end{array}$ & $\begin{array}{l}\text { Rocha, R. P. S., Valim, M. D., } \\
\text { Oliveira, J. L. C., Ribeiro, A. C. }\end{array}$ & $\begin{array}{c}\text { Pesquisa } \\
\text { transversal, } \\
\text { quantitativa }\end{array}$ & 2019 \\
\hline $\begin{array}{l}\text { 9. Estresse ocupacional da equipe de } \\
\text { enfermagem de um serviço de atendimento } \\
\text { móvel de urgência. }\end{array}$ & $\begin{array}{l}\text { Meireles, A. R., Machado, M. G., } \\
\text { Silva, R. M., Santos, O. P., } \\
\text { Moraes-Filho, I. M., Ribeiro, F. M. } \\
\text { S. S. }\end{array}$ & $\begin{array}{l}\text { Estudo transversal, } \\
\text { analítico e } \\
\text { quantitativo }\end{array}$ & 2018 \\
\hline $\begin{array}{l}\text { 10. Anxiety symptoms and quality of } \\
\text { interaction among oncology nurses: a } \\
\text { correlational, cross-sectional study }\end{array}$ & $\begin{array}{l}\text { Maria, N. K.., Karaniola, M., } \\
\text { Giannakopoulou, M., Kalafati, M., } \\
\text { Kaite, C. P., Patiraki, E., } \\
\text { Mpouzika, M., Papathanassoglou, } \\
\text { E. E.D., Middletonn. }\end{array}$ & $\begin{array}{l}\text { Pesquisa descritiva, } \\
\text { transversal, } \\
\text { correlaconal }\end{array}$ & 2016 \\
\hline $\begin{array}{l}\text { 11. Estresse ocupacional em profissionais de } \\
\text { enfermagem }\end{array}$ & $\begin{array}{l}\text { Llapa-Rodriguez, E. O., Oliveira, J. } \\
\text { K. A., Neto, D. L., Gois, C. F. L., } \\
\text { Campos, M. P. A., Mattos, M. C. } \\
\text { T. }\end{array}$ & $\begin{array}{c}\text { Pesquisa descritiva, } \\
\text { correlacional e } \\
\text { quantitativa }\end{array}$ & 2018 \\
\hline $\begin{array}{l}\text { 12. Prevalence of stress and Burnout syndrome } \\
\text { in hospital nurses woring in shifts }\end{array}$ & $\begin{array}{l}\text { Bezerra, C. M. B., Silva, K. K. M., } \\
\text { Costa, J. W. S., Farias, J. C., } \\
\text { Martino, M. M. F., Medeiros, S. M. }\end{array}$ & $\begin{array}{l}\text { Pesquisa descritiva, } \\
\text { analítica }\end{array}$ & 2019 \\
\hline $\begin{array}{l}\text { 13. Estresse ocupacional e fatores associados } \\
\text { entre enfermeiros de hospitais públicos. }\end{array}$ & $\begin{array}{l}\text { Scholze, A. R.,Martins, J. T., } \\
\text { Robazzi, M.L.C.C., Haddad., } \\
\text { M.C.F.L., Galdino, M. J. Q., } \\
\text { Ribeiro, R. P. }\end{array}$ & $\begin{array}{l}\text { Pesquisa transversal } \\
\text { analítica-descritiva }\end{array}$ & 2017 \\
\hline
\end{tabular}




\begin{tabular}{|c|c|c|c|}
\hline $\begin{array}{l}\text { 14. Percepção do estresse e estressores de } \\
\text { enfermeiros de um hospital universitário. }\end{array}$ & Silva,J.M., Malagris,L.E. N. & $\begin{array}{l}\text { Pesquisa descritiva, } \\
\text { transversal e } \\
\text { quantitativa }\end{array}$ & 2019 \\
\hline $\begin{array}{l}\text { 15. O corpo fala: aspectos físicos e psicológicos } \\
\text { do estresse em profissionais de enfermagem }\end{array}$ & $\begin{array}{c}\text { Rodrigues, C. C. F. M., Santos, V. } \\
\text { E. P. }\end{array}$ & $\begin{array}{l}\text { Pesquisa } \\
\text { quantitativa de } \\
\text { abordagem } \\
\text { descritiva } \\
\end{array}$ & 2015 \\
\hline $\begin{array}{l}\text { 16. Estresse e burnout em profissionais de } \\
\text { enfermagem de unidade de terapia intensiva } \\
\text { e semi-intensiva. }\end{array}$ & $\begin{array}{l}\text { Silva, G. S.A., Silva, G. A. V., } \\
\text { Silva, R. M., Andolhe, R.; Padilha, } \\
\text { G., Costa, A. L. S. }\end{array}$ & $\begin{array}{l}\text { Pesquisa analítica, } \\
\text { transversal e } \\
\text { quantitativa }\end{array}$ & 2018 \\
\hline $\begin{array}{l}\text { 17. Prevalência da síndrome metabólica entre } \\
\text { trabalhadores de enfermagem e associação } \\
\text { com estresse ocupacional, ansiedade e } \\
\text { depressão. }\end{array}$ & $\begin{array}{l}\text { Ribeiro, R. P., Marziale, M. H. P., } \\
\text { Martins, J. T., Ribeiro, P. H. V., } \\
\text { Robazzi, M. L. C. C.,Dalmas, J. C. }\end{array}$ & $\begin{array}{l}\text { Estudo descritivo, } \\
\text { correlacional }\end{array}$ & 2015 \\
\hline
\end{tabular}

Fonte: Autores.

Identificou-se que a população estudada pelos autores dos estudos, correspondeu, principalmente, àqueles da área hospitalar. Destaca-se que apenas 30\% dos estudos foram realizados em hospitais universitários e a maioria na rede pública.

Das evidências científicas selecionadas, a maioria dos estudos buscaram evidências sobre o estresse no ambiente de trabalho dos profissionais de enfermagem (94\%). Apenas $29 \%$ dos estudos investigaram a ansiedade nos profissionais e $17 \%$ a depressão. Cabe mencionar que, apenas $23 \%$ das pesquisas evidenciaram mais de uma doença (23\%) nos profissionais. O Quadro 2 apresenta a descrição dos artigos encontrados nas bases de dados, de acordo com o tamanho amostral, local da pesquisa, a ansiedade, o estresse e a depressão.

Quadro 2 - Distribuição dos estudos em relação a ansiedade, estresse e depressão. (n = 17), Brasil, 2020.

\begin{tabular}{|c|c|c|c|c|c|}
\hline \multirow{2}{*}{ Artigo } & \multirow{2}{*}{$\begin{array}{l}\text { Tamanho } \\
\text { amostral }\end{array}$} & \multirow{2}{*}{ Local de pesquisa } & Ansiedade & Estresse & Depressão \\
\hline & & & $\%$ & $\%$ & $\%$ \\
\hline 1 & 37 & $\begin{array}{l}\text { Hospital público do } \\
\text { Rio de Janeiro }\end{array}$ & - & 64,9 & \\
\hline 2 & 309 & $\begin{array}{l}\text { Hospital geral de } \\
\text { Prado Valadares. }\end{array}$ & - & 20,8 & - \\
\hline 3 & 43 & $\begin{array}{c}\text { Hospital de Santa } \\
\text { Maria }\end{array}$ & 11,63 & 62,7 & 9,3 \\
\hline 4 & 24 & $\begin{array}{c}\text { Hospital privado do } \\
\text { oeste do Paraná }\end{array}$ & - & 87,2 & - \\
\hline 5 & 40 & $\begin{array}{l}\text { Hospital da polícia } \\
\text { militar do Rio de } \\
\text { Janeiro }\end{array}$ & - & 25 & - \\
\hline 6 & 18 & $\begin{array}{c}\text { Hospital público em } \\
\text { Barra do Garças/ } \\
\text { MT }\end{array}$ & 55,55 & 77,77 & - \\
\hline 7 & 287 & $\begin{array}{l}\text { Hospital público de } \\
\text { alta complexidade } \\
\text { de São Paulo }\end{array}$ & - & 46,13 & - \\
\hline 8 & 104 & $\begin{array}{c}\text { Hospital } \\
\text { universitário } \\
\text { público do Centro- } \\
\text { oeste }\end{array}$ & - & 62,2 & - \\
\hline
\end{tabular}


Research, Society and Development, v. 10, n. 1, e2210111295, 2021

(CC BY 4.0) | ISSN 2525-3409 | DOI: http://dx.doi.org/10.33448/rsd-v10i1.11295

\begin{tabular}{|c|c|c|c|c|c|}
\hline 9 & 22 & $\begin{array}{c}\text { Serviço de } \\
\text { Atendimento Móvel } \\
\text { de Urgência e }\end{array}$ & - & 45,5 & - \\
\hline 10 & 72 & $\begin{array}{l}\text { Hospital grego de } \\
\text { Oncologia }\end{array}$ & 11,1 & - & - \\
\hline 11 & 101 & $\begin{array}{c}\text { Hospital } \\
\text { universitário do } \\
\text { estado de Sergipe }\end{array}$ & - & 54 & - \\
\hline 12 & 108 & $\begin{array}{c}\text { Hospital } \\
\text { Universitário }\end{array}$ & - & 36 & - \\
\hline 13 & 185 & $\begin{array}{c}\text { Três Hospitais } \\
\text { públicos do Paraná }\end{array}$ & - & 55,6 & - \\
\hline 14 & 70 & $\begin{array}{c}\text { Unidades do } \\
\text { Hospital } \\
\text { Universitário } \\
\text { Clementino Fraga } \\
\text { Filho } \\
\end{array}$ & - & 67 & - \\
\hline 15 & 21 & $\begin{array}{c}\text { Unidade de Terapia } \\
\text { Intensiva do } \\
\text { Hospital } \\
\text { Universitário em } \\
\text { Natal/ RN } \\
\end{array}$ & - & 57,1 & - \\
\hline 16 & 50 & $\begin{array}{c}\text { Hospital } \\
\text { Universitário de } \\
\text { São Paulo }\end{array}$ & 44 & 48 & 12 \\
\hline 17 & 226 & $\begin{array}{c}\text { Hospital } \\
\text { universitário }\end{array}$ & 68,1 & 27 & 81,8 \\
\hline
\end{tabular}

Fonte: Autores.

Ao analisar o nível de evidência dos estudos selecionados, por meio da proposta de Gershon e colaboradores (1999), constatou-se que os estudos apresentaram fracos níveis de evidência, uma vez que todos os estudos tiveram abordagem descritiva, considerada como nível 6 de evidência. Entre os estudos analisados, não foram encontradas pesquisas de ensaio clínicos, estudos de coorte ou caso-controle, que possuem níveis de evidência mais fortes, conforme Quadro 3. 
Quadro 3 - Distribuição dos estudos em relação à classificação do nível de evidência das publicações. $(\mathrm{n}=17)$, Brasil, 2020.

\begin{tabular}{|l|c|c|c|}
\hline \multicolumn{1}{|c|}{ Tipo de estudo } & $\begin{array}{c}\text { Nível de } \\
\text { evidência* }\end{array}$ & N \\
\hline $\begin{array}{l}\text { Meta-análise, Revisão Sistemática de Ensaio Clínico } \\
\text { Randomizado, Ensaio Clínico Randomizado Controlado }\end{array}$ & 1 & 0 \\
\hline $\begin{array}{l}\text { Pelo menos um Ensaio Clínico Randomizado Controlado bem } \\
\text { delineado }\end{array}$ & 2 & 0 & 0 \\
\hline Ensaio Clínico, sem randomização & 3 & 0 & 0 \\
\hline Estudo de Coorte, Estudo Caso-controle & 4 & 0 & 0 \\
\hline Revisão Sistemática de estudos descritivos ou qualitativos & 5 & 0 & 0 \\
\hline Estudo descritivo ou qualitativo & 6 & 17 & 100 \\
\hline Opinião de autoridades ou Relatório de Comitê de Especialistas & 7 & 0 & $\mathbf{1 0 0}$ \\
\hline
\end{tabular}

*Gershon, et al., 1999; Goyatá et al., 2016. Fonte: Autores.

\section{Discussão}

Buscou-se por meio desta revisão, analisar as evidências dos estudos que investigaram a ansiedade, o estresse e a depressão entre profissionais de enfermagem. Assim, foi possível observar a maior parte dos estudos que atenderam aos critérios de inclusão, foram publicados entre 2018 e 2019. Isso mostra o quanto esse tema tem sido investigado entre os estudos, demonstrando a sua relevância no meio científico e uma problemática ainda atual entre os profissionais de enfermagem.

Nestes anos, observou-se predomínio de estresse moderado entre os trabalhadores de enfermagem, sendo o alto nível de estresse predominante em técnicos de enfermagem. Os sinais e sintomas de estresse mais frequentes na equipe de enfermagem são sentimento de desgaste e sobrecarga de trabalho, seguido por desvalorização, conflito de funções e condições de trabalho, gerando o estresse ocupacional (Silva et al., 2018).

O estresse ocupacional está relacionado a forma como o trabalhador percebe seu ambiente de trabalho, normalmente o encarando como ameaçador e extenuante, sem perspectiva de melhora ou com cobranças excessivas e abusivas. Essa percepção ameaçadora pode ser de cunho profissional e/ou pessoal, assim o profissional acredita não possuir recursos adequados ou suficiente para enfrentá-las (Silva, 2016).

A consequência do estresse ocupacional consiste em prejuízo na interação do profissional com o ambiente de trabalho ou com o trabalho em si. Ele também pode ser entendido como manifestações psico-orgânicas de desequilíbrio, descompensação ou perda da homeostase, que ocorrem quando a experiência ou a percepção de clima organizacional se torna desfavorável, combinando vários fatores e dimensões (Monteiro, 2007). 
Dos estudos analisados nesta investigação, constatou-se que a maioria foram realizados em instituições hospitalares e públicas. Chama-se atenção para a quantidade de estudos realizados em hospitais universitários. Esta situação pode ser justificada pelo fato da maior parte das pesquisas serem realizadas em universidade públicas, que são parceiras de instituições de saúde para o desenvolvimento de pesquisas. Alguns estudos reafirmam esta justificativa, por demonstrarem a realização de pesquisas em hospitais públicos e universitários.

O estudo de Rocha e colaboradores (2020) foi realizado em um hospital universitário público de uma capital do Centro Oeste brasileiro, devido a instituição ser referência em atenção de pré-natal de alto risco, em triagem neonatal, com alta demanda de trabalho dos profissionais de enfermagem. O estudo de Silva \& Malagris, 2019 foi realizado em um Hospital Universitário, devido ao fato dos profissionais de enfermagem possuírem grande demanda de trabalho, alto nível de estresse e situações ambientais estressoras.

Evidenciou-se nesta pesquisa, que a maioria dos estudos buscaram evidências sobre o estresse no ambiente de trabalho dos profissionais de enfermagem. Apenas uma pequena porcentagem de estudos investigou a ansiedade e a depressão nos profissionais. Este fato chama atenção para o quanto o estresse é investigado no contexto de trabalho da enfermagem, demonstrando sua importância enquanto doença ocupacional presente nas instituições de saúde.

O processo de trabalho que a equipe de enfermagem desenvolve no ambiente hospitalar, pode ser passível de estresse ocupacional. Isso se deve, em decorrência de fatores estressores como: o estresse provocado por pacientes e familiares, pela própria equipe, pela morte de um paciente, pela falta de compreensão e colaboração de pacientes, a sobrecarga de trabalho em decorrência do processo, entre outros (Teixeira et al., 2017).

Outro fator que pode gerar estresse no trabalho da enfermagem é a fragilidade no relacionamento entre os membros da equipe, que pode deixar o trabalhador abalado e sensível. Este fator pode estar associado ao desgaste e a sobrecarga de trabalho, bem como a limitação de recursos humanos e materiais. Outros fatores, considerados como subjetivos, também podem causar estresse no trabalho da enfermagem, como a falta de comunicação, os conflitos com a chefia, as relações com os pacientes e familiares e a dificuldade no gerenciamento da equipe (Araújo et al., 2016).

Além disso, vale salientar a relação entre a autonomia e responsabilidade, em que o aumento de responsabilidade, decorrente do aumento da autonomia, pode gerar mais cargas de trabalho e maior tensão entre os trabalhadores, provocante estresse. A percepção de apoio social do trabalho, confiança no grupo e ajuda na realização das tarefas por parte de colegas e superiores, poderia atuar como fator de proteção/amortecimento dos efeitos negativos provocados pelo estresse e pelos trabalhos de alta exigência (Oliveira et al., 2018).

Cabe mencionar o limitado número de estudos que buscaram investigar a ansiedade e a depressão dos trabalhadores da enfermagem. Essas são doenças que também estão presentes, recorrentemente, no ambiente de trabalho dos profissionais de enfermagem e que precisam ser mais investigadas.

Algumas situações dentro do ambiente de trabalho dos profissionais de enfermagem podem provocar a ansiedade, destacando-se entre inúmeras circunstâncias, a instabilidade ou agravamento do estado de saúde dos pacientes, falta de material, de equipamentos e de pessoal, relacionamento com familiares do paciente, assim como as dificuldades para a sistematização da assistência de enfermagem e os procedimentos de alta complexidade (Silva et al., 2016).

Estudos nacionais e internacionais, apontam uma incidência elevada de ansiedade entre os profissionais de enfermagem, demonstrando que entre 30 e $70 \%$ dos profissionais possuem sinais e sintomas de transtorno de ansiedade (Barros et al., 2018; Cheung \& Yip 2016).

Com relação a depressão é uma variável de fundamental importância entre os estudos da saúde mental e da saúde dos trabalhadores, estima-se que, por ser uma doença do futuro, quase $20 \%$ da população passarão por, pelo menos, um episódio de 
depressão ao longo da vida (Pessini \& Bertachini, 2006). Essa situação pode colocar em risco a prática de enfermagem e a qualidade da assistência prestada aos usuários.

A depressão prejudica em muito as atividades laborais do profissional de enfermagem, pois dentre outros fatores já citados, causa ainda uma grande insatisfação no trabalho e na vida, intensificando os conflitos com a equipe. A convivência em equipe pode se tornar insustentável, levando o profissional a buscar uma recolocação em outra equipe, o que normalmente pouco ajudará no tratamento da doença (Pereira et al., 2017).

Ao analisar o nível de evidência dos estudos, constatou-se que os estudos apresentaram fracos níveis de evidência, pois todos enquadraram-se no nível 6 de evidência. Este fato demonstra a constante construção metodológica de estudos observacionais ou a adoção de abordagens descritivas, que buscam descrever a situação/condição em apenas um momento. Deste modo, percebe-se a escassez e necessidade de investigações com abordagens de ensaio clínicos, estudos de coorte ou caso-controle, que possuem níveis de evidência mais fortes.

Contudo, a qualidade de uma evidência pode ser alterada por alguns fatores. As evidências encontradas nos ensaios clínicos randomizados e controlados apresenta alta qualidade, mas, podem ser passíveis de diminuição dessa qualidade, dependendo do seu desenho metodológico e tamanho amostral. Enquanto isso, alguns estudos observacionais, podendo também aumentar ou diminuir sua qualidade, isso vai depender de alguns fatores presentes no estudo, como o tamanho amostral e seu delineamento metodológico. Os estudos observacionais são estudos de baixa qualidade, porém em situações pouco usuais eles podem apresentar-se com moderada ou alta qualidade (Guyatt et al., 2008a, Guyatt et al., 2008b).

A classificação da qualidade da evidência pode sofrer variações de acordo com o instrumento utilizado e o avaliador. Contudo, por meio da combinação de instrumentos, pode se determinar a classificação da evidência de uma forma mais clara, forte e objetiva. Desta maneira, pode se dizer que um alto nível de evidência não sugere, necessariamente, uma forte recomendação do estudo, bem como uma forte recomendação pode ser feita de um estudo com nível de evidência mais baixo. (Guyatt et al., 2008a, Guyatt et al., 2008b).

Na prática baseada em evidências é considerada a qualidade do estudo, por meio dos sistemas de classificação das evidências que, são qualificados de forma hierárquica, variando de acordo com a abordagem metodológica do estudo. Na prática médica baseada em evidências, os sistemas de classificação consideram somente pesquisas quantitativas. Já na enfermagem baseada em evidência, são considerados, pelos sistemas de classificação, os estudos que apresentam abordagens qualitativas e quantitativas (Galvão, 2006).

Este estudo apresentou algumas limitações, como o delineamento metodológico, que não permitiu uma maior compreensão e analise dos fatores de ansiedade, estresse e depressão entre os profissionais de enfermagem. Além disso, a busca na literatura também foi outro fator limitante, devido a quantidade reduzida de artigos que abordaram a temática investigada.

Nesse sentido, sugere-se a realização de outras investigações, que busquem compreender amplamente a causa e efeito do estresse, da ansiedade e da depressão em profissionais de enfermagem, como por exemplo, os estudos com nível de evidência forte, como os ensaios clínicos, estudos de coorte ou caso-controle.

\section{Conclusões}

Evidenciou-se que, a reduzida produção científica existente sobre transtornos mentais em profissionais de enfermagem apresentou um nível de evidência fraco. Ademais, a enfermagem revelou-se como uma profissão de risco para o estresse ocupacional, sofrendo diversas influências relacionadas à organização e ao processo de trabalho. 
Este fato, demonstra o quanto ainda precisam ser investigados os transtornos mentais na enfermagem, principalmente, por meio de estudos com abordagens que apresentem maiores níveis de evidência. Espera-se que este estudo possa contribuir para a compreensão do tema e ser motivo para novas pesquisas, devido ao número reduzido de estudos relacionados aos transtornos mentais e a enfermagem.

\section{Referências}

Alves, A. P. (2015). Prevalência de transtornos mentais comuns entre profissionais de saúde [Prevalence of common mental disorders among health professionals]. Revista Enfermagem UERJ, 23(1), 64-69. https://doi.org/10.12957/reuerj.2015.8150

Andrade, J. V. et al (2019). Ansiedade, um dos problemas do século XXI. Revista de Saúde de ReAGES, Paripiranga (BA), 2(4), $34-39$.

Araujo, M. P. S., Medeiros, S. M., Quental, L. L. C. (2016). Relacionamento interpessoal da equipe de enfermagem: fragilidades e fortalezas. Revista de Enfermagem da UERJ, 24(5),7657. http://www.facenf.uerj.br/v24n5/v24n5a09.pdf.

Barros, F. C. et al (2018). Transtornos de ansiedade em profissionais de enfermagem de serviços oncológicos. 2018. http://www.congresso2018.abrasme.org.br/resources/anais/8/1519877152_ARQU IVO_TranstornosdeAnsiedadeemprofissionaisdeenfermagem.pdf.

Borges Teixeira, L., Portela Veloso, L. U., Pereira Ribeiro, Í. A., Nunes de Oliveira, T., \& Leal Cortez, A. C. (2017). Estresse ocupacional na enfermagem atuante na unidade de terapia intensiva. Investigación En Enfermería: Imagen y Desarrollo, 19(2), 195. https://doi.org/10.11144/javeriana.ie19-2.eoea

Carlotto, M. S (2017). Transtornos mentais comuns em trabalhadores de unidades básicas de saúde: prevalência e fatores associados. Psicologia Argumento, 34 (85). https://doi:10.7213/psicol.argum.34.085.AO04.

Carvalho, D. B. de, Araújo, T. M. de, \& Bernardes, K. O. (2016). Transtornos mentais comuns em trabalhadores da Atenção Básica à Saúde. Revista Brasileira de Saúde Ocupacional, 41(0). https://doi.org/10.1590/2317-6369000115915.

Cheung, T., \& Yip, P. S. F. (2016). Self-harm in nurses: prevalence and correlates. Journal of Advanced Nursing, 72(9), $2124-2137$. https://doi.org/10.1111/jan.12987

Fernandes, D. M., \& Marcolan, J. F. (2017). Trabalho e sintomatologia depressiva em enfermeiros da Estratégia de Saúde da Família. SMAD. Revista Eletrônica Saúde Mental Álcool e Drogas (Edição Em Português), 13(1), 37. https://doi.org/10.11606/issn.1806-6976.v13i1p37-44

Galvão, C. M. (2006). Níveis de evidência. Acta Paulista de Enfermagem, 19(2), 5-5. https://doi.org/10.1590/s0103-21002006000200001

Gershon, R. R. M., Karkashian, C. D., Vlahov, D., Kummer, L., Kasting, C., Green-McKenzie, J., Escamilla-Cejudo, J. A., Kendig, N., Swetz, A., \& Martin, L. (1999). Compliance With Universal Precautions in Correctional Health Care Facilities. Journal of Occupational \& Environmental Medicine, 41(3), 181189. https://doi.org/10.1097/00043764-199903000-00007

Goyatá, Sueli Leiko Takamatsu. (2016.). Efeitos da acupuntura no tratamento da ansiedade: revisao integrativa. Revista Brasileira de Enfermagem, 69(3), 602-609. https://doi.org/10.1590/0034-7167.2016690325i

Guyatt, G. H., Oxman, A. D., Kunz, R., Falck-Ytter, Y., Vist, G. E., Liberati, A., \& Schünemann, H. J. (2008). Going from evidence to recommendations. BMJ, 336(7652), 1049-1051. https://doi.org/10.1136/bmj.39493.646875.ae

Guyatt, G. H., Oxman, A. D., Kunz, R., Vist, G. E., Falck-Ytter, Y., \& Schünemann, H. J. (2008). What is "quality of evidence” and why is it important to clinicians? BMJ, 336(7651), 995-998. https://doi.org/10.1136/bmj.39490.551019.be

Mendes, K. D. S., Silveira, R. C. de C. P., \& Galvão, C. M. (2008). Revisão integrativa: método de pesquisa para a incorporação de evidências na saúde e na enfermagem. Texto \& Contexto - Enfermagem, 17(4), 758-764. https://doi.org/10.1590/s0104-07072008000400018 
Research, Society and Development, v. 10, n. 1, e2210111295, 2021 (CC BY 4.0) | ISSN 2525-3409 | DOI: http://dx.doi.org/10.33448/rsd-v10i1.11295

Oliveira, E. B., Gallasch, C. H., Silva Junior, P. P. A., Oliveira, A. V. R., Valério, R. L., \& Dias, L. B. S. (2017). Estresse ocupacional e burnout em enfermeiros de um serviço de emergência: a organização do trabalho [Occupational stress and burnout in nurses of an emergency service: the organization of work]. Revista Enfermagem UERJ, 25(0). https://doi.org/10.12957/reuerj.2017.28842

Pereira, I. F., Faria, L. C., Vianna, R. S. M., Corrêa, P. D. S., Freitas, D. A., \& Soares, W. D. (2017). Depressão e uso de medicamentos em profissionais de enfermagem. Arquivos de Ciências Da Saúde, 24(1), 70. https://doi.org/10.17696/2318-3691.24.1.2017.544

Pessini, L., \& Bertachini, L. (2006). Nuevas perspectivas en cuidados paliativos.Acta Bioethica, 12(2). https://doi.org/10.4067/s1726-569x2006000200012

Rocha, R. P. S., Valim, M. D., De Oliveira, J. L. C., \& Ribeiro, A. C. (2020). Características do trabalho e estresse ocupacional entre enfermeiros hospitalares. Enfermagem Em Foco, 10(5). https://doi.org/10.21675/2357-707x.2019.v10.n5.2581

Rosmarie Hajjar. (2017). Depressão e a busca do "Pharmakon" para aplacar o mal estar individual e social. Revista Família, Ciclos de Vida e Saúde No Contexto Social, 5(0), 165-174. https://doi.org/10.18554/refacs.v5i0.2004.

Santana, L. L., Sarquis, L. M. M., Brey, C., Miranda, F. M. D., \& Felli, V. E. A. (2016). Absenteísmo por transtornos mentais em trabalhadores de saúde em um hospital no sul do Brasil. Revista Gaúcha de Enfermagem,37(1). https://doi.org/10.1590/1983-1447.2016.01.53485

Santos, A. S., Monteiro, J. K., Dilélio, A. S., Sobrosa, G. M. R., \& Borowski, S. B. V. (2017). Contexto hospitalar público e privado: impacto no adoecimento mental de trabalhadores da saúde. Trabalho, Educação e Saúde, 15(2), 421-438. https://doi.org/10.1590/1981-7746-sol00054

Silva, Durval Veloso da. (2017). Ansiedade, estresse, depressão e uso de drogas entre trabalhadores de enfermagem no ambiente hospitalar. http://doi.org/10.14393/ufu.di.2017.365

Silva, G. S. A., Silva, G. A. V., Silva, R. M., Andolhe, R., Padilha, K. G., Costa, A. L. S (2018). Estresse e burnout em profissionais de enfermagem de unidade de terapia intensiva e semi-intensiva. Revista de Divulgação Científica Sena Aires. 7(1), 5-11.

Silva, J. M., \& Malagris, L. E. N. (2019). Percepção do estresse e estressores de enfermeiros de um hospital universitário. Estudos e Pesquisas Em Psicologia, 19(1), 71-88. https://doi.org/10.12957/epp.2019.43007

Silva, N. C. et al (2016). Transtornos à saúde mental relacionados à intensa rotina de trabalho do enfermeiro: uma revisão bibliográfica. Revista Eletrônica Estácio Saúde. 5(2), 107-122.

Souza, M. T. de, Silva, M. D. da, \& Carvalho, R. de. (2010). Integrative review: what is it? How to do it? Einstein (São Paulo), 8(1), 102-106. https://doi.org/10.1590/s1679-45082010rw1134

Teixeira, L. B. et al (2017). Estresse ocupacional na enfermagem atuante na unidade de terapia intensiva. Investigación en Enfermería: Imagen y Desarrollo, 19 (2), 195-211. 